\title{
Grammatische Entwicklungstendenzen der deutschen Gegenwartssprache - ein Einblick
}

\author{
Grammatical Developmental Tendencies in Contemporary German - \\ an Insight
}

\section{ZUSAMMENFASSUNG}

Dieser Beitrag gibt anhand von Beispielen einen Einblick in grammatische Entwicklungstendenzen der deutschen Gegenwartssprache. Nach einer Diskussion zentraler Begriffe (wie Entwicklungstendenzen) werden unterschiedliche Tendenzen beschrieben.

Schlüsselwörter: grammatische Entwicklungstendenzen, deutsche Gegenwartssprache, Kasusmarkierung, Kasuswechsel, Tendenzen im Verbal- und syntaktischen Bereich

\section{ABSTRACT}

This paper gives an insight into grammatical developmental tendencies in contemporary German by way of examples. After a discussion of important technical terms (like developmental tendencies), several tendencies are described.

Keywords: grammatical developmental tendencies, contemporary German, casus marking, casus change, verbal and syntactic tendencies

\section{Einleitung}

Sprachwandel ist nicht nur ein Phänomen der Vergangenheit, sondern auch der Gegenwart, da Sprache niemals statisch ist. Eine Sprachgemeinschaft verändert, (seltener) willentlich (durch Sprachpolitik und Sprachplanung) oder (zumeist) unwillentlich, die aktuelle Sprache in Gebrauch und/oder auch in der Norm und/ oder gegebenenfalls sogar im System. Jede Sprecherin, jeder Sprecher ist damit durch ihr/sein Sprechen einer Sprache an Sprachwandel beteiligt - und daran, dass Sprache nicht homogen, sondern vielfältig ist und auf verschiedenen Ebenen in Gebrauch, Norm und System variiert. Der folgende Beitrag möchte die aktuelle Variation und Vielfalt in der Grammatik des Gegenwartsdeutschen exemplarisch an besonders auffälligen Beispielen unter dem Begriff der „Entwicklungstendenzen“ in den Blick nehmen. Zuvor werden zentrale Begrifflichkeiten geklärt.

Christian Efing, Institut für Sprach- und Kommunikationswissenschaft, Rheinisch-Westfälische Technische Hochschule Aachen, Eilfschornsteinstraße 15, 52062 Aachen, c.efing@isk.rwth-aachen.de, https://orcid.org/0000-0002-2488-6171 


\section{Begrifflichkeiten}

\subsection{Gegenwartssprache}

Unter Gegenwartssprache soll im vorliegenden Beitrag mit Bär (2000) das Deutsche ab ca. 1950 verstanden werden. Diese Einordnung entspricht nicht nur - ein wenig gewollt und zufällig - der traditionellen Einteilung der deutschen Sprachgeschichte in Perioden von grob 300 Jahren $^{1}$, sondern sie trägt auch Entwicklungen ab der Nachkriegszeit ${ }^{2}$ Rechnung, deren sprachliche Konsequenzen Bär zusammenfasst mit den Stichworten

- „Öffnung der Grenze zwischen Varietäten und Standardsprache“ (S. 22) (Rückgang der Dialekte, Verbreitung von Fachsprachen),

- „Ausgleich zwischen gesprochener und geschriebener Sprache“(S. 24f) (Elemente gesprochener Sprache werden in der Schriftlichkeit aufgenommen),

- „Ausgleich der Stilebenen“ („Tendenz zum allgemeinsprachlichen Verzicht auf stilistisch gehobene Varianten“, „Aufwertung ehemals als niedrig empfundener Varianten") (S. 25).

Bär (2000) konstatiert, dass sich im Deutschen unter diesen ,veränderten historischen, kulturellen und kommunikationstechnischen Bedingungen“"Veränderungen in den Bereichen Morphologie, Lexik, Syntax und im Textbereich ergeben haben, die das Ansetzen einer neuen Sprachperiode ab 1950 sinnvoll erscheinen lassen. Nach Erscheinen des Beitrags von Bär haben die Entwicklungen in den so genannten Neuen Medien mit ihren sprachlich-kommunikativen Konsequenzen und neuen Kommunikationsformen (E-Mail, [WhatsApp-]Chat usw.) die bereits von Bär angesprochenen drei Entwicklungen sicherlich noch verstärkt und vor allem das Eindringen mündlicher Phänomene in die mediale Schriftlichkeit weiter deutlich befördert.

\subsection{Dimensionen der Variation}

Um die Phänomene der Variation innerhalb einer Einzelsprache zu beschreiben und zu kategorisieren, haben sich in der varietäten- und soziolinguistischen vier bis fünf Dimensionen herauskristallisiert, die einander jedoch keineswegs ausschließen. Konkret sind dies traditionell die diachrone (Variation in der Zeit), die diatopische (Variation im Raum, z.B. Dialekte, Regiolekte, nationale Varietäten), die diastratische (Variation aufgrund sozialer Faktoren wie Schicht, Alter usw.) und die diaphasische (Variation in Abhängigkeit von Situation und Funktion) Dimension, die neuerdings häufig durch eine diamediale (Variation

1 750-1050 Althochdeutsch, 1050-1350 Mittelhochdeutsch, 1350-1650 Frühneuhochdeutsch, ab 1650 Neuhochdeutsch.

2 Ausgelöst durch gesellschaftliche Entwicklungen der Egalisierung (sozialer Schichten und ihres Sprachgebrauchs), des Engagements (in Studenten-, Frauen-, Friedensbewegung usw.) und der Emanzipation, im Bereich der (Massen-)Medien, der europäischen Integration und der Globalisierung. 
in Abhängigkeit vom Medium) ergänzt werden. Der hier im Beitrag gewählte Begriff der Entwicklungstendenzen verweist deutlich auf eine Verortung der zu beschreibenden Phänomene in der diachronen Dimension. Jedoch muss die Herkunft der sich verbreitenden Phänomene oft auf der diatopischen, diastratischen oder diamedialen Ebene gesucht werden.

\subsection{Sprachwandel vs. Entwicklungstendenzen}

Wenn in diesem Beitrag von Entwicklungstendenzen die Rede ist und nicht von aktuell sich vollziehendem Sprachwandel, dann wird der Begriff mit Braun (1998, S. 93) so verwendet, dass es sich bei den beschriebenen Phänomenen um aktuelle Tendenzen handelt, deren Prozess der Entwicklung nicht abgeschlossen ist und von denen daher auch noch nicht prognostizierbar ist, ob sich diese Phänomene halten und (gar in der Standardsprache) durchsetzen werden.

Daher liegt ein weiterer Begriff ganz in der Nähe: der der sprachlichen Zweifelsfälle (vgl. etwa Klein, 2018). Wenn eine Sprache sich in Norm und/oder System im Begriff ist zu verändern, treten alte und neue Formen parallel auf. Damit kommt es sprecherseitig, aber überindividuell in der Sprachgemeinschaft zu Unsicherheiten, welche Sprachform synchron (in der Standardvarietät) als die korrekte oder angemessene, welche (noch) als Fehler oder (schon) als akzeptabel gilt. Insbesondere die Sprachöffentlichkeit sowie die nicht-linguistische öffentliche Sprachkritik (vgl. etwa die Zwiebelfisch-Kolumne von Bastian Sick: https:// bastiansick.de/category/kolumnen/zwiebelfisch/) sieht hier Neuerungen oft aus einer präskriptiven Warte und verurteilt sie als Fehler und Sprachverfall, während die Linguistik die Neuerungen in Form und Funktion beschreibt und ggf. anhand der Beschreibung von Verwendungshäufigkeiten, -funktionen und -kontexten versucht, Entwicklungstendenzen zu erkennen und Entwicklungsprozesse zu beschreiben und zu begründen. Die folgende Grafik verdeutlicht, wie sich ad hoc auftretende Phänomene in einem Prozess der Konventionalisierung „durch Fakten" vom Sprachgebrauch kommend durchsetzen können; sie zeigt aber auch, dass die betreffenden Phänomene wieder verschwinden können.

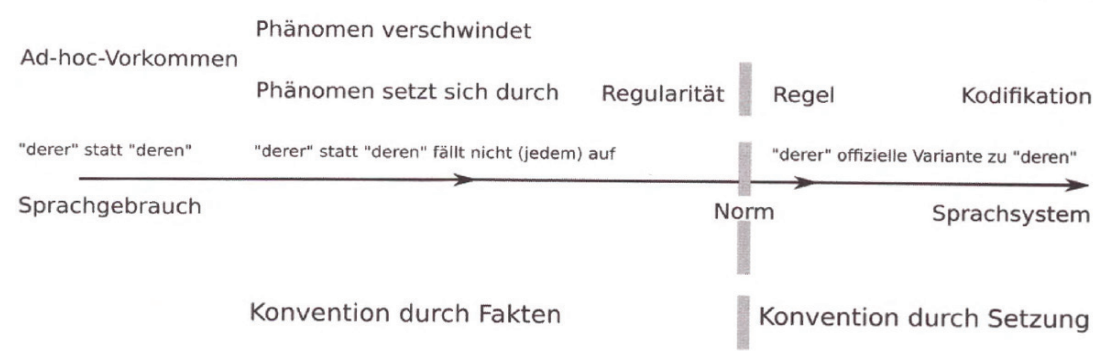

Abbildung 1: Das Kontinuum Ad-hoc-Vorkommen - Regularität-Regel-Kodifikation im Spiegel von Sprachgebrauch - Sprachnorm - Sprachsystem (Felder, 2016, S. 41) 
Entwicklungstendenzen sind diese aus dem Sprachgebrauch heraus kommenden neuen Phänomene, die noch nicht zur Regularität oder gar kodifizierten Regel geworden sind und die daher noch vor der Entscheidung stehen, ob sie sich in die Standardvarietät hinein durchsetzen oder ob sie eventuell gar wieder verschwinden.

Man wird bei vielen der im Folgenden beschriebenen Phänomene einen Ursprung vor 1950 nachweisen und damit sehen, dass viele der angeblich aktuellen Entwicklungstendenzen keine neuen Erscheinungen sind, sondern sich nur jetzt erst - ggf. medial schriftlich und bis in die öffentliche Kommunikation hinein in die Richtung Alltagskommunikation verbreiten - aus regionalen oder sozialen Sprechergemeinschaften heraus, zum Teil befördert durch die so genannten Neuen Medien. Ein Hinweis darauf bzw. Indikator dafür, ob oder dass sich die Phänomene durchsetzen und zur Regularität werden, wäre ein zunehmendes Eindringen auch in Kontexte der konzeptionellen Schriftlichkeit.

\section{Grammatische Entwicklungstendenzen der deutschen Gegenwartssprache}

Man könnte den Beitrag nun im Folgenden gliedern nach der vermutlichen Herkunft der Entwicklungstendenzen: So ist die Ausbreitung der tun-Periphrase und des am-Progressivs ebenso auf eine dialektale Herkunft rückführbar wie das Phänomen, Personennamen mit neutralem Genus zu verwenden (das Merkel, das Gauck); die Verbreitung der Auslassung von Artikeln und Präpositionen (Isch geh Kino.) etwa lässt sich auf ethnolektale Ursprünge zurückführen. Im hier vorgelegten Beitrag aber wird ein sprachsystematisches Vorgehen gewählt, das exemplarische Entwicklungstendenzen entlang der linguistischen Dimensionen beschreibt. Insbesondere werden dabei morphologische Phänomene im Nominalbereich, syntaktische Entwicklungstendenzen sowie das Tempus- und Modussystem in den Blick genommen. Lexikalische (z.B. Anglizismen) wie semantische, (ortho-)graphische (z.B. Emojis) wie textuelle Phänomene (z.B. Kommunikationsformen, Textsorten und Multimodalität in social media) bleiben dabei aus Platzgründen ebenso ausgespart wie Entwicklungstendenzen in den Varietäten und Registern des Deutschen (Entwicklung der Fachsprachen, von sog. leichter oder einfacher Sprache usw.).

\subsection{Kasuswahl und Kasusmarkierung bei Maskulina}

Im Folgenden wird hier der Kasus-Bereich speziell am Beispiel zweier Entwicklungstendenzen hervorgehoben: 1) der (angebliche) Rückgang des Genitivs, den insbesondere der Sprachkritiker Bastian Sick mit seiner Buchreihe Der Dativ ist dem Genitiv sein Tod ins Licht der deutschen Öffentlichkeit gerückt und nicht unbedingt zurecht kausal mit einem angeblichen Erstarken des Dativs ins Verbindung gebracht hat, sowie 2) Deklinationsklassenwechsel bei den schwachen Maskulina. 


\subsubsection{Kasuswahlverschiebungen}

Einer der (Hinter-)Gründe, warum Sick im Titel seiner Buchreihe ein Erstarken des Dativs als Ursache für einen Genitivrückgang ausmacht, darf vermutlich in dem Paradebeispiel schlechthin ausgemacht werden, das die sprachinteressierte Öffentlichkeit in Deutschland immer wieder und seit Jahrzehnten diskutiert: den partiellen Kasuswechsel bei der Präposition wegen, die traditionell den Genitiv regiert, die aber schon seit geraumer Zeit oft mit dem Dativ verwendet wird, was aber von vielen Sprachteilhabern immer noch als stilistisch unschön empfunden wird, auch wenn das Phänomen bereits 1986 im Rechtschreib-Duden erwähnt wurde.

Der Kasus erfüllt verschiedene Funktionen- wie etwa die Beziehungen innerhalb eines Satzes zu verdeutlichen oder die Interpretation bestimmter Präpositionalphrasen zu erleichtern - und wird entweder durch Rektion (bei Verben, Adjektiven, Nomen, Präpositionen), Kongruenz (die Nominalphrase erhält den Kasus von einer Bezugs-Nominalphrase: Er bemächtigte sich [der Festung] als [des wichtigsten Zugangs zum Pass].) oder semantische Kasuszuweisung (die Nominalphrase erhält den Kasus aufgrund ihrer Bedeutung im Satz): Sie trat [festen Schrittes] in den Ring) ausgelöst. Nach Braun (1998, S. 111) gibt es im Deutschen 31 Grundformen von Satzmodellen mit je unterschiedlicher Häufigkeit. Dabei sind Veränderungen v.a. an 4 Positionen zu beobachten:

1) Subjekt - Prädikat - Genitivobjekt $\rightarrow$ Rückgang

2) Subjekt - Prädikat - Dativobjekt $\rightarrow$ Rückgang

3) Subjekt - Prädikat - Akkusativobjekt $\rightarrow$ Zunahme

4) Subjekt - Prädikat - Präpositionalobjekt $\rightarrow$ Zunahme

Diese Veränderungen, die sich also nicht zugunsten des Dativs, sondern des Akkusativs und der Präpositionalobjekte vollziehen, stehen in einem Wechselverhältnis: die Zunahme von 3) und 4) bedingt Abnahme von 1) und 2). In allen Fällen haben wir es mit Bewegungen im Bereich der Verbrektion zu tun.

Der Genitiv befindet sich demnach nur als Genitivobjekt auf dem Rückzug; es gibt nur noch 14 Verben mit Genitiv-Objekt (sich annehmen, sich bedienen, bedürfen, sich befleißigen, gedenken, sich rühmen ...), die zumeist als veraltend oder förmlich und fern der Alltagssprache angesehen werden und in Dialekten und in der Umgangssprache auch wohl nie verbreitet waren. Stabil ist der Genitiv hingegen in festen Wendungen: sich eines Besseren besinnen, seines Amtes walten, das spottet jeder Beschreibung, das entbehrt jeder Grundlage, guter Dinge sein, viel Aufhebens, meines Wissens, unseres Erachtens (S. 111).

Daneben stehen Verben mit schwankender Rektion: jemandes/eines Dinges achten - auf jdn./etw. achten; sich einer Sache erinnern - sich an eine Sache erinnern; sie spottet seiner - sie spottet über ihn; er besinnt sich seiner Kraft-auf seine Kraft; er klagt ihn des Diebstahls an - wegen Diebstahl.

Es ist jedoch auch in anderen Bereichen ein Genitiv-Rückgang zu konstatieren, so bei prädikativen Adjektiven (begierig, kundig, eingedenk, mächtig, voll), die 
mittlerweile oft mit der Präposition von verwendet werden, sowie bei bestimmten Präpositionen (kraft, behufs, betreffs, um ...willen, wegen, hinsichtlich, bezüglich, angesichts), bei denen tatsächlich ein Ersatz durch den Dativ zu beobachten ist. Bisweilen wirkt hier die Genitivverwendung schon manieriert ('wegen Urlaubs geschlossen; 'Wegen dieses Mistes rufst Du mich an?), sodass man konstatieren kann, dass in solchen Fällen der Dativ auch im Geschriebenen bereits als üblich gelten kann (Glück \& Sauer, 1997, S. 49-52).

Umgekehrt weitete sich in gewissen Kontexten der Genitiv jedoch auch aus - zum Teil auf Kosten des Dativs, wie bei einigen Präpositionen (gemäß, entgegen, nahe trotz). Insbesondere (v.a. nachgestellte) Genitivattribute sind nach wie vor sehr beliebt, weiten sich im Amtsdeutsch eventuell sogar aus und sind auch im Gesprochenen resistent gegenüber einer Ablösung durch Präpositionalausdrücke mit von, und auch der prädikative Genitiv (ich bin deiner Meinung) ist stabil.

Die Tendenzen der Akkusativierung und Präpositionalisierung sind unter anderem zu erklären durch die größere Ökonomie und Funktionsleichtigkeit (Braun, 1998, S. 113):

- Präpositionen differenzieren Verbaussagen genauer (statt einheitlicher Artikel): achten auf, anklagen wegen, sich erinnern an. Ein Problem ist jedoch, dass Präpositionen zum Teil die Merkmale der Orts- und Richtungsangabe aufgegeben haben (in warten auf etw. hat auf nur eine figurativ-idiomatische Bedeutung).

- Eine formale Vielfalt bedeutet einen Verlust an Ökonomie und eine größere Anstrengung für die Sprachfähigkeit und das Gedächtnis, was ein Argument insbesondere für Fremdsprachenlerner ist.

Die Akkusativierung ist rückführbar auf eine zunehmende Umwandlung von Verbbeständen und vor allem das Vordringen von Verben mit be-Präfix (ornative Verben: jdm. einen Zuschuss geben $>j d n$. bezuschussen), aber auch auf andere Präfixe (privative Verben: jdm. den Mut nehmen > jdn. entmutigen) (S. 114).

Während die Tendenz zur Präpositionalisierung der grundlegenden Sprachwandeltendenz vom Synthetischen zum Analytischen entspricht, ist die Akkusativierung als Kasus-Substitution ein Indiz dafür, dass die Kasuswahl als arbiträr bzw. semantisch unmotiviert empfunden wird.

Ein neues, offenbar noch unbeschriebenes und zu erforschendes Phänomen, das dem Verfasser dieses Beitrags seit wenigen Jahren bei der Korrektur studentischer Arbeiten von Deutsch-Erstsprachler*innen auffällt, ist die systematische Verwendung des Dativs ab der zweiten Position in Aufzählungen, die eigentlich den Genitiv oder Akkusativ erfordern (Typ: ich sehe den Tisch, dem Stuhl...); aber auch generell an Stellen, an denen die Kasuskongruenz und der Bezug unklar scheinen, etwa in Appositionen, wird auf den Dativ ausgewichen, wie in: 
- „Betrachtet werden Varianten der Flexion von Fremdwörtern, dem Genus bei der Integration von Fremdwörtern und der Pluralbildung von Fremdwörtern".

- „Somit kann behauptet werden, dass es sich bei diesem Ausdruck nicht nur um Kategorie (VI), sondern auch um Kategorie (III), dem CodeSwitching, handelt".

Dem Wechsel von Genitiv zu Dativ in Beispiel 1 (,dem Genus“) wird vermutlich eigentlich ein Wechsel von Genitiv zu einer Präpositionalkonstruktion zugrunde liegen, bei angenommenem elliptischem von (,Varianten der Flexion... und [von] dem Genus").

Unklar ist die Interpretation im Falle von Appositionen, in denen gehäuft der Wegfall der Kasusmarkierung oder aber eher der Übergang zum Nominativ zu konstatieren ist wie in folgendem Beispiel: dass von 20 Jugendlichen (DeutscheØ und AusländerØ) nur zwei einen Fahrschein entwerten.

\subsubsection{Kasus- (und Plural-)Markierung}

Ein anderer Fall liegt vor, wenn nicht der Kasus wechselt, sondern der Kasus anders als standardmäßig markiert oder flektiert wird. Hierunter fällt einerseits die an die umgangssprachliche Aussprache (phonologische Reduktion) angelehnte reduzierte Verschriftung des Akkusativs (Kein[en] Schutt abladen!; Mach kein [en] Scheiß, zit. nach Glück \& Sauer, 1997, S. 53). Aber auch der Dativ wird schriftlich bisweilen nicht mehr normgemäß markiert: mit neuem PräsidentØ, seinem PilotØ; zum bloßen ErfüllungsgehilfeØ. Hier wie in anderen Fällen auch im Akkusativ (Die Prinzessin küsst den Prinz; ich habe einen Bär gesehen) liegt entweder ein Markierungsverzicht oder ein Flexionsklassenwechsel im Sinne einer Vereinheitlichung und Vereinfachung vor, der dann funktional erscheint, wenn formalen Differenzierungen, die eine Sprache komplexer machen, „keine inhaltlichen Differenzierungen gegenüberstehen“, sondern die formalen Differenzierungen „grammatischen Ballast" bedeuten (Wegener, 2007, S. 43). Gehäuft fehlt die Kasusmarkierung bei schwachen Maskulina, wo dieses Fehlen dann als Flexionsklassenwechsel (von schwach zu stark) interpretiert wird.

Mit Wegener (2007) ist die Ko-Existenz starker und schwacher Maskulina „dann kein grammatischer Ballast, wenn diese Klassen dem Hörer zusätzliche Information vermitteln“ (S. 43), und diese zusätzliche Information ist eine semantische, da der Flexionsklassenwechsel als semantische Remotivierung der schwachen Maskulina zu interpretieren ist, denn es wird ein Hinweis auf das semantische Merkmal [+/- belebt] gegeben: Schwache Maskulina tragen fast immer das Merkmal [+belebt] (vgl. S. 43), sodass ein Wechsel belebter Maskulina aus der gemischten Deklinationsklasse (z.B. bei Autor) in die Klasse der schwachen Maskulina eine semantische Motivation hat. 
Dennoch sind gerade im Bereich der schwachen Maskulina viele Normverletzungen zu beobachten, wenn schwache Maskulina abwandern und wie starke behandelt werden. Die Gruppe der schwachen Maskulina zeigt hier einerseits Abbautendenzen, wenn in Dativ und Akkusativ Singular die Deklinationsendungen entfallen (Die Prinzessin küsst den PrinzØ, dem BärØ); andererseits gibt es aber eben auch „Belege für Aufbauprozesse“ (Köpcke, 2005, S. 68-69) - offenbar insbesondere bei fremden Maskulina und Neutra auf -or (die Meinung von dem Autoren; vgl. auch Thieroff, 2003).

Köpcke (2005) erarbeitet eine Prototypikalitätsskala für schwache Maskulina, anhand derer die Abbautendenzen erklärbar sind:

\begin{tabular}{llllll} 
Abbildung (3): & \multicolumn{2}{c}{ Prototypikalitätsskala für die schwachen Maskulina } & prototypisch \\
prototypisch \\
schwach
\end{tabular}

Abbildung 2: Prototypikalitätsskala für schwache Maskulina (Köpcke, 2005, S 72)

Prototypisch für schwache Maskulina sind demnach Mehrsilber mit Pänultimabetonung, finalem Schwa-Laut und dem semantischen Merkmal [+menschlich]. Je weiter rechts auf der Skala eine Form anzusiedeln ist, umso größer ist die Wahrscheinlichkeit, dass die Deklinationsmarkierungen der schwachen Maskulina entfallen. Prinz als Einsilber und Bär als Einsilber mit dem semantischen Merkmal [-menschlich] sind demnach keine prototypischen schwachen Maskulina und deswegen anfällig für Markierungsabbau und Kandidaten für einen Flexionsklassenwechsel, wie Köpcke (2005, S. 76-77) in Untersuchungen unter Schülern und Studierenden auch empirisch nachweisen konnte.

Da die normalerweise gemischt flektierenden Maskulina auf -or zumeist aber ebenso die Merkmale des linken Pols der Prototypikalitätsskala für schwache Maskulina aufweisen, nämlich Mehrsilbigkeit und das semantische Merkmal [+menschlich], und da zudem die Pluralbildung auf -en erfolgt und im Plural eine Pänultimabetonung vorliegt (Autóren also analog wie bei Matrósen), ist empirisch gestützt (S. 79) zu vermuten, dass diese Ähnlichkeitsrelationen die Ursache für die geänderten Kasusmarkierungen (des Autors $>$ des Autoren) bzw. für den Flexionsklassenwechsel hin zu den schwachen Maskulina sind. 
Kasus(markierungs)wechsel sind größtenteils also nicht willkürlich und bedingt durch Unsicherheiten (Thieroff, 2003), sondern sie sind zumeist funktional und systematisch interpretierbar als „Tendenzen zur Regularisierung und damit zur Vereinfachung“ (Wegener, 2007, S. 47), am Beispiel der Flexionsklassenwechsel etwa von schwacher zu starker (des Funken/des Funkens) oder von gemischter zu schwacher (des Autors/Autoren) Flexion.

Insbesondere der von der Sprachkritik bemängelte Wegfall von Kasusendungen (Rückkehr zum PlanetØ der Affen) scheint auch aus anderem Grunde „systemangemessen“ (S. 48) und funktional, da man schwache Akkusativ- oder Dativ-Singularformen in artikellosen Kontexten nicht als solche erkennen kann, sondern als Plural interpretiert, etwa wenn „ein Wort auf Konsonanten endet, ein Orchester mit Dirigenten auftritt“" (S. 48; vgl. aus der Presse auch Ägyptischer Polizist schießt Christen nieder [Bild, 12.1.2011], Stichwahl entscheidet über Präsidenten in Nordzypern [Die Welt, 17.4.2000]). Heute wird der Kasus oft durch den Artikel markiert (zu Grabe tragen > zum Grab tragen), was im Genitiv zum Beispiel auch zu Redundanzen führt (der Preis des Wassers ), die zum Teil abgebaut werden (der Bau des Potsdam-CenterØ) (Die Welt, 17.4.2000]). Im Bereich des Genitivs sind Kasusmarkierungsänderungen (des Bubs statt des Buben; des Markgrafs statt des Markgrafen) einerseits durch die Prototypikalität des $-s$ als Genitivmarker, andererseits aber auch durch den Erhalt der Silbenstruktur bei -s-Marker zu erklären.

Wegener prognostiziert insgesamt einen Verlust der Markierung des Dativ Singular, nicht aber des Genitiv Singular und Dativ Plural. Für die letzten beiden Fälle geht sie vielmehr von einer ,Aufspaltung der deutschen Sprache in Schriftdeutsch und Umgangsdeutsch“ aus, „dass sich also zwei Systeme, zwei Grammatiken herausbilden werden. $\mathrm{Zu}$ zahlreich sind die Kontexte, in denen es ohne Kasussuffix zu uneindeutigen Phrasen kommt bzw. käme“" (Wegener, 2007, S. 50). Die gesprochene Sprache ginge dann zur präpositionalen Lösung über, die es auch bereits im Geschriebenen für Stoffsubstantive und Pluralformen gibt (der Preis von Wasser) (S. 50).

Weitere Entwicklungstendenzen können aus Platzgründen nur noch summarisch genannt, aber nicht mehr ausführlich behandelt werden.

\subsection{Verbalbereich}

\subsubsection{Konjugation}

Im Verbalbereich ist die sicherlich bekannteste und schon jahrhundertelang währende Entwicklung die des Umbaus von Verben starker Konjugation hin zu schwacher (er boll > er bellte; er molk > er melkte). Gegentendenzen gibt es weit seltener: so wechselte fragen auch schon einmal von der schwachen in die starke Konjugation - und dann wieder zurück; der Übergang des Partizips von winken (von gewinkt > gewunken) ist ein Prozess, der nur diese Form und nicht das gesamte winken-Paradigma betrifft. 
Für einen Wechsel zur schwachen Konjugation scheinen die Imperativformen besonders anfällig, so sind in der gesprochenen Sprache Imperative wie Ess(e)! (statt Iss!), Les(e)! (statt Lies!), Helf mir! (statt Hilfmir!) bereits weit verbreitet und sie dringen auch in die geschriebene Sprache ein. Partizipien wechseln hingegen als letzte Form (ich melkte statt ich molk, aber immer noch ich habe gemolken statt ich habe gemelkt, vgl. etwa Bittner \& Köpcke, 2008).

Auch wenn starke Verben tendenziell zurückgehen, so halten sie sich dennoch stabil im hochfrequenten Bereich, sodass ihr gänzliches Verschwinden nicht anzunehmen ist. Wegener (2007, S. 37) betont hierzu mit Blick auf das stärkere Differenzierungspotenzial der starken Verben: „Es ist kein Luxus, im Hochfrequenzbereich deutlich zu differenzieren, es ist funktional. [...] Eine Sprache mit einem gewissen Anteil irregulärer Formen funktioniert besser." Zum Beispiel ist die Unterscheidung von (sie) gehen - gingen erheblich deutlicher und besser erkennbar als die zwischen (sie) suchen-suchten.

Ebenfalls stark in der Sprachöffentlichkeit diskutiert ist die Verwendung des Vollverbs brauchen ohne $z u$ ( $d u$ brauchst das nicht $\varnothing$ machen) in verneinten oder einschränkenden Sätzen mit einem Infinitiv, das in dieser semantischen Bedeutung in syntaktischer Analogie zum synonymen Modalverb müssen ( $d u$ musst das nicht machen) gebildet wird. In der gesprochenen Sprache ist diese Tendenz der Verwendung von brauchen in der Bedeutung von ,müssen“ als und wie ein Modalverb bereits weitgehend verbreitet und wird gestützt durch Elision der Personalendungen in der 1. und 3. Person Singular in der gesprochenen Sprache (ich brauch $\varnothing$, du brauchst, er brauch Ø), die den regulären Endungen der Modalverben (ich muss $\varnothing$, du musst, er muss $\varnothing$ ) entsprechen (Lehmann, o.J.).

\subsubsection{Tempus}

Im Tempusbereich fallen vor allem zwei Entwicklungstendenzen ins Auge, die das Entstehen bzw. die Verbreitung zweier neuer Tempora in breitere Bereiche des Sprachgebrauchs betreffen. Das erste sich neu entwickelnde Tempus ist der am-Progressiv, auch Rheinische Verlaufsform genannt (ich bin am Lesen). Progressivformen sind in anderen germanischen Sprachen wie dem Englischen und Niederländischen längst etabliert und könnten das deutsche Tempussystem in Form einer Grammatikalisierung sinnvoll erweitern, jedoch gibt es klare regionale Präferenzen (Vorkommen v.a. in West- und Südwestdeutschland) und syntaktische Beschränkungen ('ich bin einen Apfel am Schälen).

Weitere zwar alte, aber neuerdings wieder aufkommende und vieldiskutierte Tempusformen sind das insgesamt recht unsystematisch und eher idiolektal verbreitete so genannte (Doppelte) Perfekt II (Ich habe den Computer runtergefahren gehabt.) und (Doppelte) Plusquamperfekt II (Er war hingefallen gewesen.) in den Funktionen etwa der Markierung von Vorvergangenheit und der Verstärkung temporaler Aspekte im modalen Kontext. 


\subsubsection{Modus}

Die auffälligste Tendenz im modalen Bereich ist sicherlich die deutliche Verbreitung der analytischen Konjunktivbildung mit würde (ich würde kommen statt ich käme), die auch eine formale Erleichterung bei weitgehend unbekannt gewordenen Formen im Zuge des Rückgangs der Konjunktiv II-Verwendung darstellt (ich würde helfen statt ich hülfe). Doch auch der Konjunktiv I befindet sich in bestimmten Kontexten auf dem Rückzug, so etwa bei markierter indirekter Rede, wo er redundant ist (Er hat gesagt, dass er heute kommt/komme.). In diesem Umfeld ist auch eine zunehmend fehlende Differenzierung zwischen Konjunktiv I und II zu beobachten und der Konjunktiv II wird bei Formgleichheit von Konjunktiv I und Indikativ bevorzugt (Peter sagt, Paul komme/käme heute bestimmt, vgl. Glück \& Sauer, 1997, S. 65-68).

Insgesamt ist die Konjunktivverwendung, generell wie in formaler Hinsicht (analytisch oder synthetisch) oft eine Frage des passenden (situativ angemessenen) oder persönlichen Stils ('Flöhest Du mit mir aus der Kneipe, hülfest Du mir.)

Eine weitere Entwicklung im Verbalbereich betrifft die Verwendung der auxiliaren tun-Periphrase (Ich tu das nie wieder machen.), die stilistisch im Gesprochenen wie Geschrieben in den meisten Positionen extrem stark markiert ist.

\subsection{Position und Flexion der Adjektive}

Zwei interessante Phänomene im Bereich der Adjektive sind das postnominale (Wahlkampf pur) und das unflektierte Adjektiv (lecker Pils) Adjektiv (vgl. Dürscheid, 2002; Stoltenburg, 2008). Nachgestellte, zumeist einsilbige Adjektive fanden bis in die 1950er Jahre v.a. nur eine poetische Verwendung (Hänschen klein), breiten sich seitdem aber auch im Gastronomiebereich (Rum pur, Forelle blau) und in den Medien (Sonne satt; Zeitschriftentitel wie TV digital) sowie seit den 1990ern auch im politischen Bereich (Kapitalismus pur) aus.

Das attributiv vorangestellte, aber unflektierte lecker scheint eine Besonderheit unter den Adjektiven zu sein, das sich in dieser unflektierten Variante größtenteils mit Nomen aus dem Bereich der Nahrungsmittel verbindet (lecker Pils trinken, macht lecker Kaffee, lecker Lachs). Diese „Sonderform im heutigen Deutsch“ (Stoltenburg, 2008, S. 150) im rein informellen Sprachgebrauch ist eventuell durch das Niederländische beeinflusst, in dem ,das Lexem lekker unflektiert in sehr unterschiedlichen pragmatischen und semantischen Kontexten gebraucht werden kann“ (S. 150).

\subsection{Tendenzen im syntaktischen Bereich}

Das meistdiskutierte Phänomen im Bereich der Syntax ist sicherlich die weit verbreitete, aber noch weitgehend auf das Gesprochene beschränkte Verbzweit-/ Hauptsatzwortstellung im Nebensatz nach den Subjunktionen weil, obwohl und während (Ich konnte nicht kommen, weil ich war krank.). Interessant ist hier eine Betrachtung des Phänomens aus funktionaler Perspektive, die deutlich macht, 
dass die geänderte Verbstellung in vielen Fällen nicht einfach eine fehlerhafte Abweichung von der Standard-Satzstellung ist, sondern dass die Verbzweitstellung erhebliche semantische und (diskurs-)pragmatische Implikationen hat und auch in gewissen syntaktischen Kontexten nicht vorkommen kann, also durchaus systematisch ist (vgl. etwa Wengel \& Efing, 2014).

Weitere Tendenzen betreffen die Ausklammerung (Er kam schneller zurück als erwartet.), die Ausgliederung (Wirklich: Das war aber nett von Klaus.) und die Abnahme von Gliedsätzen bei Zunahme von Nominalgruppen etwa im Bereich der Verwaltungssprache.

Im Kontext der Kommunikation in Neuen Medien dringt die Auslassung/ Einsparung von Artikel, Subjekt und Objekt als Form einer situativen und strukturellen Ellipse verstärkt in die mediale Schriftlichkeit ein (Muss erst Computer heile machen), ebenso die Verwendung von Inflektivkonstruktionen (*wildmitdenarmenwink*).

\section{Ausblick}

Die in diesem Beitrag exemplarisch angeführten grammatischen Entwicklungstendenzen der deutschen Gegenwartssprache sind nicht nur aus linguistischer Perspektive interessant, sondern auch aus (fremd-)sprachendidaktischer sowie mit Blick auf die öffentliche Meinung und die Sprach- und Normbewusstheit innerhalb einer Sprachgemeinschaft. Die beschriebenen Phänomene vermitteln einen wichtigen Einblick in die Vielfalt, Heterogenität und Wandelbarkeit bzw. Dynamik des Gegenwartsdeutschen auch auf der Ebene von Sprachnorm und Sprachsystem - und insbesondere in die unterschiedlichen Normen und den unterschiedlichen Sprachgebrauch in Mündlichkeit vs. Schriftlichkeit. Damit können Sprachteilhaber durch die Reflexion der bewusst gemachten Phänomene die Funktionalität der Neuerungen erkennen und die Perspektive der öffentlichen Sprachkritik hinterfragen lernen, Entwicklungstendenzen seien Fehler und ein Indiz für Sprachverfall. Gleichzeitig lernen Sprachteilhaber, ihre eigenen sprachlichen Normabweichungen einzuordnen und ihre Teilhabe an Sprachwandel und der Verbreitung neuer Phänomene zu erfahren.

Zum bewussten Beherrschen einer (Fremd-)Sprache gehört dabei insbesondere das Wissen bzw. die Varietäten- und Registerkompetenz (Efing \& Sander, im Ersch.), welche Sprachformen von wem (in welchem Alter, in welcher Region) in welchem Medium und in welcher Situation verwendet werden - und inwieweit und ggf. wann es angemessen ist, diese Non-Standard-Formen auch selbst produktiv zu verwenden. Dies bedeutet einen Einblick zu haben in die Existenz verschiedener Normengeltungsbereiche sowie in die Tatsache, dass auch NonStandard-Normen ihre eigene Berechtigung und Funktion haben und Standardabweichungen daher oftmals nicht als Fehler zu gelten haben, sondern nur nach der Kontextangemessenheit und angemessenen Registerwahl zu beurteilen sind und 
generell gesprochene Sprache spezifischen und anderen Bedingungen und Normen unterliegt als Schriftsprache. Dabei entsteht idealerweise eine Bewusstheit über den Unterschied zwischen wirklichen individuellen sprachsystematischen Fehlern, zwischen Zweifelsfällen der deutschen Sprachgemeinschaft und zwischen bereits etablierten (neuen) Sprachformen.

Die Vielfalt des Deutschen und die geographische Verteilung der verschiedenen Sprachformen des Non-Standards kann dabei gut von jedermann online anhand der Karten des Atlas zur deutschen Alltagssprache (AdA) (vgl. https:/www.atlasalltagssprache.de/) nachvollzogen werden. Stärker Sprachinteressierte können diesen Einblick vertiefen durch einen online-Besuch bei der Variantengrammatik des Standarddeutschen für das Geschriebene (vgl. http://mediawiki.ids-mannheim. de/VarGra/index.php/Start).

\section{References}

Bär, J. A. (2000). Deutsch im Jahr 2000. In K. M. Eichhoff-Cyrus, \& R. Hoberg (Eds.), Die deutsche Sprache zur Jahrtausendwende. Sprachkultur oder Sprachverfall? (pp. 9-34). Mannheim, Wiesbaden: Dudenverlag.

Bittner, A., \& Köpcke, K.-M. (2008). Sprachwandel- oder Verlotterungsprozesse - Versuch einer Versachlichung. In M. Denkler, S. Günthner, W. Imo, J. Macha, D. Meer, B. Stoltenburg, \& E. Topalovic (Eds.), Frischwärts und unkaputtbar. Sprachverfall oder Sprachwandel im Deutschen (pp. 59-80). Münster: Aschendorff.

Braun, P. (1998). Tendenzen in der deutschen Gegenwartssprache. Sprachvarietäten (4th. ed.). Stuttgart, Berlin, Köln: Kohlhammer.

Dürscheid, Ch. (2002). „Polemik satt und Wahlkampf pur“ - Das postnominale Adjektiv im Deutschen. Zeitschrift für Sprachwissenschaft, 21(1), 57-81.

Efing, Ch., \& Sander, I.-L. (in press). Registersensibilität als Reflexion eigenen und fremden kommunikativen Verhaltens. In F. Busch, P. Droste, \& E. Wessels (Eds.), Reflexivität kommunikativer Praktiken. Neue Perspektiven auf Metakommunikation. Stuttgart: Metzler.

Elspaß, S., \& Möller, R. (2003-). Atlas zur deutschen Alltagssprache (AdA). Retrieved December 12, 2020, from www.atlas-alltagssprache.de.

Felder, E. (2016). Einführung in die Varietätenlinguistik. Darmstadt: Wissenschaftliche Buchgesellschaft.

Glück, H., \& Sauer, W. W. (1997). Gegenwartsdeutsch (2nd. ed.). Stuttgart, Weimar: Metzler.

Klein, W. P. (2018). Sprachliche Zweifelsfälle im Deutschen. Theorie, Praxis, Geschichte. Berlin, Boston: De Gruyter.

Köpcke, K.-M. (2005). „Die Prinzessin küsst den Prinz“ - Fehler oder gelebter Sprachwandel? Didaktik Deutsch, 18, 67-83.

Lehmann, Ch. (n.d.). Brauchen wird zum Modalverb. Retrieved November 13, 2020, from http:// www.christianlehmann.eu/fundus/Deutsch/Dt_brauchen2Modalverb.html.

Sick, B. (2004-2015). Der Dativ ist dem Genitiv sein Tod [Book series]. Retrieved November 10, 2020, from https://bastiansick.de/category/kolumnen/zwiebelfisch/.

Stoltenburg, B. (2008). „lecka pilsken trinken!“ Deutsche Adjektive in der Umgangssprache - das Ende der Endungen? In M. Denkler, S. Günthner, W. Imo, J. Macha, D. Meer B. Stoltenburg, \& E. Topalovic (Eds.), Frischwärts und unkaputtbar. Sprachverfall oder Sprachwandel im Deutschen (pp. 129-152). Münster: Aschendorff.

Thieroff, R. (2003). Die Bedienung des Automatens durch den Mensch. Deklination der schwachen Maskulina als Zweifelsfall. Linguistik online, 16(4), 105-117. 
Wegener, H. (2007). Entwicklungen im heutigen Deutsch - Wird Deutsch einfacher? Deutsche Sprache, 1, 35-62.

Wengel, P., \& Efing, Ch. (2014). ,...weil die Verbstellung hat Funktion...” Funktion und Normengeltungsbereiche der Verbstellung im Nebensatz reflektieren. Deutsch, 5-10(41), 26-29. 